JGG 2021;69:289-291

doi: $10.36150 / 2499-6564-N 463$
Clinical Geriatrics - Reviews

\section{Lipid control in geriatric DM patients}

\author{
Claudio Pedone \\ Unit of Geriatrics, Campus Bio-Medico University, Rome, Italy; Italian Society of Gerontology and \\ Geriatrics, Florence, Italy
}

\section{RECOMMENDATIONS}

A. The cardiovascular risk profile, including the lipid profile, should be assessed in all DM patients. The frequency of follow-up examinations should be adjusted to the characteristics of the patient, particularly their functional status and life expectancy.

B. Prevention of cardiovascular complications in geriatric DM patients should be based on a risk profile derived from standard algorithms and adapted to the characteristics of the patient and their life expectancy based on a comprehensive geriatric assessment.

C. Lifestyle interventions (diet, physical activity) should be recommended and tailored to the patient's functional and nutritional status. For patients with good functional status and life expectancy statin therapy, if necessary, should be indicated as secondary prevention.

D. In patients with good functional status and life expectancy who already take statins as a primary prevention, it is appropriate to continue treatment, especially if the patient has a high cardiovascular risk. Treatment initiation with statins as a primary prevention must be carefully assessed on the basis of cardiovascular risk and as part of an overall assessment of the patient's treatment.

Received: July 30, 2021

Accepted: September 16, 2021

\section{Correspondence}

Claudio Pedone

Unit of Geriatrics, Campus Bio-Medico University, via Alvaro del Portillo 21, 00128 Rome, Italy.

E-mail: c.pedone@unicampus.it

How to cite this article: Pedone C. Lipid control in geriatric DM patients. Journal of GerontologyandGeriatrics2021;69:289-291. https://doi.org/10.36150/2499-6564-N463

(C) Copyright by Società Italiana

di Gerontologia e Geriatria (SIGG)

\section{(c) (1) $(9)$}

\section{OPEN ACCESS}

This is an open access article distributed in accordance with the CC-BY-NC-ND (Creative Commons Attribution-NonCommercial-NoDerivatives 4.0 International) license. The article can be used by giving appropriate credit and mentioning the license, but only for non-commercial purposes and only in the original version. For further information: https://creativecommons.org/licenses/by-nc-nd/4.0/deed.en
E. In patients with good functional status and life expectancy, use of ezetimibe, PCKS9 inhibitors, fibrates, and icosapent ethyl for treatment of hypercholesterolemia not controlled by statins alone or severe hypertriglyceridemia may be considered.

F. Pharmacological interventions must be prescribed in frail or functionally-compromised patients taking into account the increased risk/benefit ratio, with particular reference to the risk of adverse effects at the muscular level and pharmacological interactions related to polypharmacy. in patients with frailty or functional impairment.

\section{STRENGTH OF THE RECOMMENDATIONS}

The quality of the evidence is moderate. Recommendations are supported by published evidence and best practice.

\section{SUPPORTING EVIDENCE}

See appendix.
G. Lipid-lowering therapy targets should not be strictly applied, especially 


\section{AREAS OF UNCERTAINTY AND FUTURE PERSPECTIVES}

Information on older persons is limited because they are systematically excluded from clinical trials and safety and effectiveness data may not always be extrapolated from younger samples. More real-life studies are needed to have a better estimate of the actual efficacy and effectiveness of lipid lowering strategies in older people. Bempedoic acid seems to hold promise as a cholesterollowering agent, but the evidence is still limited.

\section{APPENDIX}

The prevention of cardiovascular complications plays a major role in the management of DM. Atherosclerotic cardiovascular disease (ASCVD) is the main cause of morbidity and mortality in people with DM. The impact of ASCVD is greater in older people: data from the Italian Higher Institute of Health (Istituto Superiore di Sanità, ISS) (https://www.epicentro.iss.it/igea/diabete/ prevalenza_comp_macro) show an incidence of cardiovascular events of 43.9/22.9 (men/women) per thousand DM patients aged 60-64, which rises to 58.4/46.5 per thousand persons aged over 75 . Older people have a greater incidence of cardiac disorders and cerebrovascular events than younger people ${ }^{1}$. Therefore, there needs to be more effective prevention of such complications in geriatric patients, while considering their individual characteristics.

Lipid-lowering therapy, together with antihypertensives, is one of the cornerstones of preventing cardiovascular complications. However, deciding on a preventive strategy must be based on reliable information on the course of the condition being prevented, the effectiveness of the intervention, and the time period during which the intervention will have the most significant impact ${ }^{2}$. Information on older persons is limited because they are systematically excluded from clinical trials. Nevertheless, there is some evidence on the effectiveness of lipid-lowering therapy in geriatric patients, although no studies have specifically focused on geriatric DM patients. In the general older population, the PROSPER trial have shown a $24 \%$ reduction of cardiovascular mortality in people treated with pravastatin ${ }^{3}$. A metaanalysis of two primary prevention trials with statins (JUPITER and HOPE) reported a $26 \%$ reduction in the combined risk of non-fatal myocardial infarction, nonfatal strokes, and cardiovascular death in a sub-group of patients aged 70 and over treated with rosuvastatin compared to patients treated with placebo ${ }^{4}$.

Similar positive results have been reported for the secondary prevention of major cardiovascular events
(stroke/TIA and major coronary events) in patients aged $\geq 65$ years who had experienced a recent cerebrovascular event ${ }^{5}$. The PROVE IT-TIMI22 trial reported that achieving target LDL cholesterol levels in patients aged $\geq 70$ with a recent acute coronary syndrome was associated with a $40 \%$ reduction in the composite risk of unstable angina, myocardial infarction, or death over two years ${ }^{6}$. These findings have been confirmed in observational studies of patients aged 75 and over ${ }^{7}$. A recent meta-analysis showed strong evidence for the effectiveness of statins for secondary prevention in older people, while there is uncertainty about the efficacy as a primary prevention strategy ${ }^{8}$.

On the basis of the above evidence, there does not seem to be an indication to start lipid-lowering therapy with statins for primary prevention in people over 75 years, while this therapy can be considered for secondary prevention in this age group. In frail patients, low doses must be used and the standard targets (LDL $<80 \mathrm{mg} / \mathrm{dl}$ or $<70 \mathrm{mg} / \mathrm{dl}$ in patients with cardiovascular disease, $\mathrm{HDL}>=40 \mathrm{mg} / \mathrm{dl}$, triglycerides $<200 \mathrm{mg} /$ di) do not need to be strictly applied.

In patients with good functional status and life expectancy in which LDL are not adequately controlled, ezetimibe or proprotein convertase subtilisin/kexin type 9 (PCSK9) inhibitors (alirocumab, evolocumab) on top of statins, or as a standalone therapy when statins are not tolerated or contrainicated, further contribute to LDL-C reduction and may be considered. In a study on patients aged $>=75$ years, ezetimibe reduced the risk of an outcome composite of sudden cardiac death, myocardial infarction, coronary revascularization, and stroke. The trial, however, was open-label, had a large number of patients lost to follow-up and was terminated early because of increment of competing risks ${ }^{9}$. With respect to PCK9-inhibitors, no data specific for older people, although a subanalysis of the ODYSSEY trial confirmed the positive effects of alirocumab in patients with age $>=65$ years ${ }^{10}$.

Always in patients in which there is a reasonable life expectancy and are in good functional status, hypertriglyceridemia associated with low HDL-cholesterol concentration may benefit from fibrates as an add-on therapy, but no evidence exists on older patients, therefore caution must be used in establishing the indication and following-up for potential side effects. In these patients the presence of hypertriglyceridemia with low LDL-concentration despite statin use may also benefit from icosapent ethyl, with the same caveats as fibrates and with special attention to the risk for atrial fibrillation and bleeding, that were slightly more frequent in the intervention group of the REDUCE-IT trial ${ }^{11}$.

Recently, bempedoic acid has become available as a lipid-lowering drug for patients who do not tolerate 
statins or do not reach target LDL cholesterol concentration despite maximized therapy with statins. The target enzyme of this drug (ATP citrate liase) is not expressed in muscular cells and this make this drug of particular interest in older people in which muscular function is frequently impaired in the context of sarcopenia. However, no information on its safety in older people is yet available, and no recommendation has been issued about its use in this population.

In conclusion, it must be emphasized that the choice of preventive strategy must take the individual patient's condition into account. The American Diabetes Association's guidelines underline that lipid-lowering therapeutic strategies must take life expectancy into account ${ }^{12}$, while the International Diabetes Federation's guidelines have specific recommendations based on the functional status of the patient (independent, dependent/frail/ dementia, terminal) ${ }^{13}$. Adapting the treatment strategy to the patient's functional status includes setting objectives as well as the methods to achieve them. For example, dietary changes should certainly be taken into consideration, but the risk of insufficient caloric intake should be avoided by monitoring dietary modifications: lipid-lowering and low sodium diets can worsen "anorexia of aging" because food loses its taste ${ }^{14}$.

Ethical consideration

None.

\section{Acknowledgement}

None.

\section{Funding}

None.

\section{Conflict of interest}

The Author declares no conflict of interest.

\section{References}

1 Huang ES, Laiteerapong N, Liu JY, et al. Rates of complications and mortality in older patients with diabetes mellitus: the diabetes and aging study. JAMA Intern Med 2014;174:251-258. https://doi.org/10.1001/ jamainternmed.2013.12956

2 Coll PP, Roche V, Olsen JS, et al. The prevention of cardiovascular disease in older adults. J Am Geriatr Soc 2020;68:1098-1106. https://doi.org/10.1111/jgs.16353
3 Shepherd J, Blauw GJ, Murphy MB, et al. Pravastatin in elderly individuals at risk of vascular disease (PROSPER): a randomised controlled trial. Lancet 2002;360:1623-1630. https://doi.org/10.1016/s0140-6736(02)11600-x

4 Ridker PM, Lonn E, Paynter NP, et al. Primary prevention with statin therapy in the elderly: new meta-analyses from the contemporary JUPITER and HOPE-3 randomized trials. Circulation 2017;135:1979-1981. https://doi. org/10.1161/circulationaha.117.028271

5 Chaturvedi S, Zivin J, Breazna A, et al. Effect of atorvastatin in elderly patients with a recent stroke or transient ischemic attack. Neurology 2009;72:688-694. https://doi. org/10.1212/01.wnl.0000327339.55844.1a

6 Ray KK, Bach RG, Cannon CP, et al. Benefits of achieving the NCEP optional LDL-C goal among elderly patients with ACS. Eur Heart J 2006;27:2310-2316. https://doi. org/10.1093/eurheartj/ehl180

7 Bezin J, Moore N, Mansiaux Y, et al. Real-life benefits of statins for cardiovascular prevention in elderly subjects: a population-based cohort study. Am J Med 2019;132:740748.e7. https://doi.org/10.1016/j.amjmed.2018.12.032

8 Ponce OJ, Larrea-Mantilla L, Hemmingsen B, et al. Lipidlowering agents in older individuals: a systematic review and meta-analysis of randomized clinical trials. J Clin Endocrinol Metab 2019;104:1585-1594. https://doi. org/10.1210/jc.2019-00195

9 Ouchi Y, Sasaki J, Arai H, et al. Ezetimibe lipid-lowering trial on prevention of atherosclerotic cardiovascular disease in 75 or Older (EWTOPIA 75): a randomized, controlled trial. Circulation 2019;140:992-1003. https://doi.org/10.1161/ circulationaha.118.039415

10 Sinnaeve PR, Schwartz GG, Wojdyla DM, et al. Effect of alirocumab on cardiovascular outcomes after acute coronary syndromes according to age: an ODYSSEY OUTCOMES trial analysis. Eur Heart J 2020;41:2248-2258. https://doi. org/10.1093/eurheartj/ehz809

11 Bhatt DL, Steg PG, Miller M, et al. Cardiovascular Risk Reduction with Icosapent Ethyl for Hypertriglyceridemia. N Engl J Med 2019;380:11-22. https://doi.org/10.1056/ NEJMoa1812792

12 American Diabetes Association. 12. Older adults: standards of medical care in diabetes, 2020. Diabetes Care 2020;43(Suppl 1):S152-S162. https://doi.org/10.2337/dc20-s012

13 International Diabetes Federation. Managing older people with type 2 diabetes: global guideline, 2013.

14 Niedert KC, American Dietetic Association. Position of the American Dietetic Association: liberalization of the diet prescription improves quality of life for older adults in longterm care. J Am Diet Assoc 2005;105:1955-1965. https:// doi.org/10.1016/j.jada.2005.10.004

\section{This statement is: \\ 冈 Recommendation (supported by published evidence) $\square$ Best practice (supported by expert opinion)}

\author{
Quality of the evidence (in the case of recommendation): \\ $\square$ Low \\ 冈 Moderate \\ High
}

